\title{
Why did the storm ex-Gaston (2010) fail to redevelop during the PREDICT experiment?
}

\author{
Thomas M. Freismuth ${ }^{1}$, Blake Rutherford ${ }^{2}$, Mark A. Boothe ${ }^{1}$, and Michael T. Montgomery ${ }^{1}$ \\ ${ }^{1}$ Naval Postgraduate School, Monterey, CA, USA \\ ${ }^{2}$ Northwest Research Associates, Redmond, WA, USA \\ Correspondence to: Michael T. Montgomery (mtmontgo@nps.edu)
}

Received: 3 September 2015 - Published in Atmos. Chem. Phys. Discuss.: 19 January 2016

Revised: 25 April 2016 - Accepted: 16 June 2016 - Published: 13 July 2016

\begin{abstract}
An analysis is presented of the failed redevelopment of ex-Gaston during the 2010 PREDICT field campaign based on the European Centre for Medium Range Weather Forecast (ECMWF) analyses. We analyze the dynamics and kinematics of ex-Gaston to investigate the role of dry, environmental air in the failed redevelopment. The flow topology defined by the calculation of particle trajectories shows that ex-Gaston's pouch was vulnerable to dry, environmental air on all days of observations. As early as 12:00 UTC 2 September 2010, a dry layer at and above $600 \mathrm{hPa}$ results in a decrease in the vertical mass flux and vertical relative vorticity. These findings support the hypothesis that entrained, dry air near $600 \mathrm{hPa}$ thwarted convective updraughts and vertical mass flux, which in turn led to a reduction in vorticity and a compromised pouch at these middle levels. A compromised pouch allows further intrusion of dry air and quenching of subsequent convection, therefore hindering vorticity amplification through vortex tube stretching. This study supports recent work investigating the role of dry air in moist convection during tropical cyclogenesis.
\end{abstract}

\section{Introduction}

Recent work has established a new overarching framework for understanding tropical cyclone formation from easterly waves (Dunkerton et al., 2009, hereafter DMW09). This framework, for describing how such hybrid wave-vortex structures develop into tropical depressions, was likened to the development of a marsupial infant in its mother's pouch. By analogy, a juvenile proto-vortex is carried along by its parent wave until the proto-vortex is strengthened into a self- sustaining entity. For tropical storms developing from within tropical waves, the recirculating flow in the wave's critical layer corresponds to the "wave-pouch". Here, the wave and mean-flow speeds are similar, along a critical latitude oriented approximately parallel to the easterly jet, and the trough axis intersects meridionally. The critical latitude is the latitude where the mean flow and wave phase speeds are equal (DMW09). Storm formation occurs typically near the intersection of critical latitude and trough axis. ${ }^{1}$

The new cyclogenesis model and accompanying scientific hypotheses were established observationally in the Atlantic and eastern Pacific sectors by DMW09. The new models find additional support in idealized numerical modeling simulations (Wang et al., 2010a, b; Montgomery et al., 2010a; Nicholls and Montgomery, 2013), recent case studies in the field in the western North Pacific during the Tropical Cyclone Structure Experiment 2008 (TCS08, Montgomery et al., 2010b; Lussier III, 2010; Montgomery et al., 2012; Raymond and López-Carrillo, 2011; Lussier III et al., 2014), in the Atlantic during the Pre-Depression Investigation of Cloud Systems in the Tropics (PREDICT) campaign in 2010 (Montgomery et al., 2012; Smith and Montgomery, 2012; Davis and Ahijevych, 2012, 2013), in NASA's ongoing Hurricane and Severe Storm (HS3) missions (2012-2016) and the case of Hurricane Sandy (Lussier III et al., 2015). The field data afford a resolved view of horizontal and vertical structure in the wave pouch and its immediate surroundings, valuable for system centering, circulation magnitude, vortic-

\footnotetext{
${ }^{1}$ The jet contains two such critical latitudes, the cyclonic one equatorward of the jet axis being instrumental to storm formation, the anticyclonic one poleward of the jet axis relevant to dusty Saharan air outbreaks and dry subsidence aloft.
} 
ity balance, interleaving of air masses, and moist thermodynamic profiles.

A corollary from the new model is that the nondevelopment of a candidate tropical disturbance is linked to the pouch structure being compromised. Currently, it is thought that there are two principal ways the pouch can be compromised. The first way is a combined kinematicdynamic effect caused by the differential shearing of the pouch in the vertical plane. The increased shear and deformation of the pouch tends to compromise the resilience of the vortex (Reasor et al., 2004) and produce a vertically misaligned distribution of moisture generated by the convection. The second way is a combined thermodynamic-dynamic effect associated with the intrusion of dry air (so-called "antifuel") into the otherwise moist pouch from a relatively dry environment. The injection of anti-fuel into the wave-pouch acts to limit the vigor of deep convection in the middle and upper troposphere and the amplification of vertical vorticity in convective updraughts above the boundary layer (Kilroy and Smith, 2012), which is essential for spinning up a tropical cyclone (Smith and Montgomery, 2012).

The non-developing case of ex-Gaston (2010) during the PREDICT experiment is arguably one of the most extensively observed non-developing tropical disturbances ever. The 5 consecutive days of observational data for such a nondeveloping disturbance is unprecedented.

Based on the foregoing discussion, there remains an important question in understanding the non-development of ex-Gaston: did ex-Gaston have a robust (closed), protective pouch? If ex-Gaston did, in fact, have a robust pouch, one would expect the system to redevelop and possibly intensify. We will show that ambient vertical shear and the entrainment of dry, environmental air early on 2 September led to the degradation of ex-Gaston's pouch and this plagued the convection within the pouch for the entire observational period of the PREDICT experiment.

\section{Review of Pre-PREDICT Gaston}

Tropical Storm Gaston developed from an African easterly wave that moved westward from the African coast on $28 \mathrm{Au}$ gust 2010. The National Hurricane Center (NHC) designated Gaston as a tropical storm at 15:00 UTC 1 September. Despite being in a favorable environment with relatively low vertical shear (discussed further below) and an SST of $28.5^{\circ} \mathrm{C}$ (Gjorgjievska and Raymond, 2014), convection associated with Gaston quickly diminished, and the NHC downgraded the system to a post-tropical/remnant low by 21:00 UTC 2 September. Convective activity increased on 3 September; however it did not re-organize and the system remained a remnant low.

\section{Data sources}

This study uses the European Centre for Medium-Range Weather Forecasts (ECMWF) analyses from 28 August to 11 September 2010. The analysis fields have a horizontal resolution of $0.25^{\circ}, 25$ vertical levels from 1 to $1000 \mathrm{hPa}$, and temporal output every $6 \mathrm{~h}$. Dropsonde data from the PreDepression Investigation of Cloud-Systems in the Tropics (PREDICT) Experiment were included in the standard assimilation system at ECMWF.

The PREDICT Experiment, as described in Montgomery et al. (2012), was a dedicated field study that set out to acquire empirical data to quantify thermodynamic and kinematic parameters in developing and non-developing tropical disturbances in the Atlantic Ocean. The primary platform for this experiment was the NSF-NCAR Gulfstream V (GV) with EOL/Vaisala GPS dropsondes. The GV was able to make drops from altitudes as high as $\sim 13 \mathrm{~km}$. There were five GV research flights with 109 dropsondes conducted during ex-Gaston (Fig. 1) ${ }^{2}$.

\section{Results}

We begin our analysis by characterizing the vertical shear that affected Gaston's pouch. The vertical shear is calculated in the vicinity of the pouch center, the center being defined here by the intersection of the wave trough and critical latitude at the $700 \mathrm{hPa}$ level. "Deep-layer shear" and "pouch shear" are computed by taking the vector differential of horizontal winds between the 200 and $850 \mathrm{hPa}$ levels, and between the 500 and $850 \mathrm{hPa}$ levels, respectively, averaged over a $3^{\circ} \times 3^{\circ}$ box centered at the pouch center (referred to as pouch-scales). The pouch-scale averaging is performed on a $3^{\circ} \times 3^{\circ}$ box, centered on the circulation center as defined by the $700 \mathrm{hPa}$ tracking level.

For both the deep and pouch shear, the magnitude of the shear decreases rapidly from $\sim 20 \mathrm{~m} \mathrm{~s}^{-1}$ on 30 August to $\sim 2 \mathrm{~m} \mathrm{~s}^{-1}$ on 2 September (Fig. 2). During the same period, the direction of the deep and pouch shear shifts from easterly to westerly flow (Fig. 3). After 2 September, the magnitude of the shear (deep and pouch) increases to $\sim 5 \mathrm{~m} \mathrm{~s}^{-1}$. The pouch shear direction slowly becomes more northerly by 5 September. The deep shear, though, rapidly changes direction from northeasterly to southwesterly from 12:00 UTC 2 September to 00:00 UTC 3 September, in the ECMWF data. The deep shear returns to an easterly flow on 4 September. These shear results are consistent with the analysis of

\footnotetext{
${ }^{2}$ There were two additional flights into ex-Gaston on 6 and 7 September with the DC- 8 from the National Aeronautics and Space Administration (NASA) as part of the Genesis and Rapid Intensification (GRIP) experiment conducted concurrently with the PREDICT experiment. The EC analysis data include both NSF and NASA dropsonde data. See Smith and Montgomery (2012) for further details.
} 


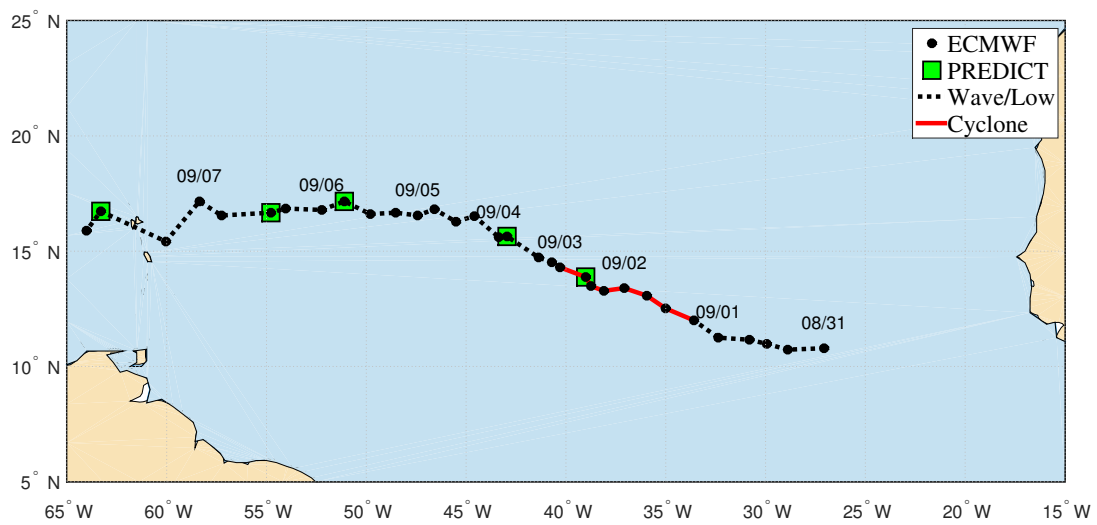

Figure 1. Track for ex-Gaston based on pouch center (black dots) as identified in the $6 \mathrm{~h}$, ECMWF analysis data. Green squares show approximate times of PREDICT research flights over the disturbance. The red line indicates when the National Hurricane Center designated the disturbance as at least a tropical depression. The black-dashed line indicates when the disturbance was an incipient wave or remnant low.

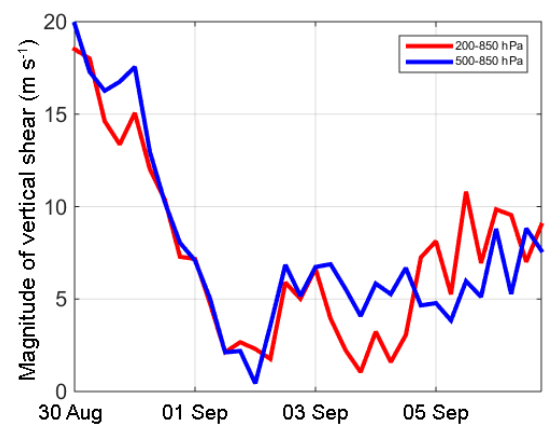

Figure 2. Magnitude of vertical wind shear. Shear is shown for the $200-850 \mathrm{hPa}$ (red line) and the $500-850 \mathrm{hPa}$ (blue line) levels. Shear decreased from $\sim 20 \mathrm{~ms}^{-1}$ on 30 August 2010 to $\sim 2 \mathrm{~ms}^{-1}$ on 2 September 2010. Although the shear is below the nominal value of $12 \mathrm{~m} \mathrm{~s}^{-1}$, there is still persistent shear on the pouch. These results are consistent with previous studies by Davis and Ahijevych (2012).

PREDICT data by Davis and Ahijevych (2012). The National Hurricane Center defines vertical shear of $12 \mathrm{~m} \mathrm{~s}^{-1}$ as an upper limit for favorable conditions for tropical cyclogenesis. The magnitude of the vertical shear (typically $4-8 \mathrm{~m} \mathrm{~s}^{-1}$ ) for ex-Gaston, while below this heuristic limit for an SST of $28.5^{\circ} \mathrm{C}$, does suggest the potential for a ventilating flow relative to the moving system (Riemer and Montgomery, 2011) and a potential contribution of a dipole-like distribution of vorticity from a non-advective flux (Haynes and McIntyre, 1987; Raymond et al., 2014). This latter contribution could be a net increase or decrease of vorticity.

The evolution of other pertinent variables is shown in Fig. 4. In the subpanels of this figure we show a time-height Hovmoeller diagram of relative humidity, relative vorticity $(\zeta)$, and vertical mass flux at each level from averages taken over a $3^{\circ} \times 3^{\circ}$ box. Similar analysis was done for a $1^{\circ} \times 1^{\circ}$ box (referred to as sub-pouch scale) centered on the circula-

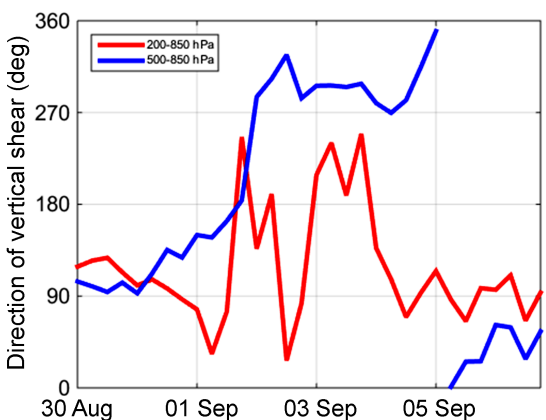

Figure 3. Direction of vertical wind shear. The direction of the wind shear is shown for the $200-850 \mathrm{hPa}$ (red line) and the 500 $850 \mathrm{hPa}$ (blue line) levels. The directions are compass directions in a meteorological sense. The $500-850 \mathrm{hPa}$ shear is mainly from the northwest on 2 September 2010. Analysis by RM12 showed that the source region for dry air was from north of the pouch.

tion center; trends were similar to those for the $3^{\circ}$ box, but are not shown. We use the model vertical velocity in pressure coordinates, $\omega$, to calculate the mass flux as $\rho w=-\omega / g$, where $\rho$ is density, $w$ is vertical velocity in height coordinates, and $g$ is the acceleration due to gravity. On both scales in the ECMWF data, a layer of dry air above $600 \mathrm{hPa}$ appears to penetrate the pouch region on 2 September, and that dry layer persists through the decline of the system (Fig. 4). Coincident with the intrusion of the dry air are system-scale decreases in relative vorticity and mass flux.

To gain insight into the apparent intrusion of dry air into the pouch beginning near 06:00 UTC 2 September (discussed above), we first examine the flow topology of exGaston using the dividing streamline methodology discussed in Riemer and Montgomery (2011). This methodology assumes for simplicity that the flow is steady in a translating frame. Although the observed flow will be shown later to have an important transient component, this technique can 

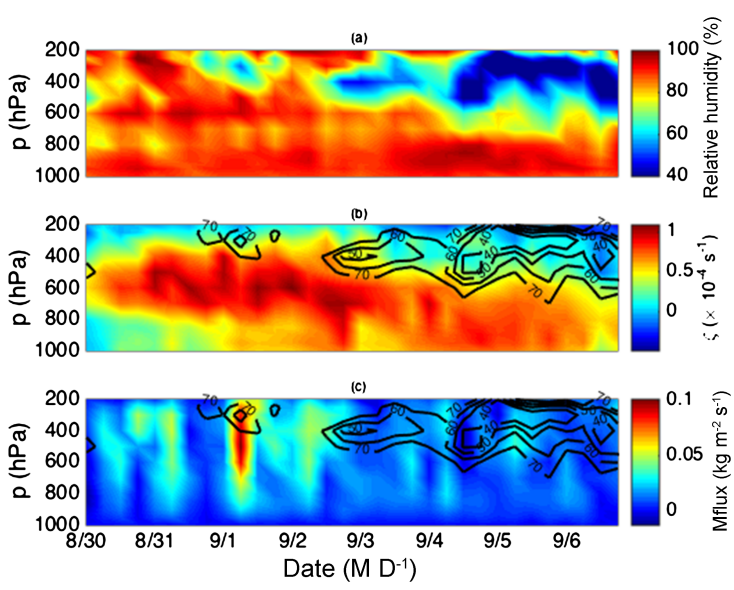

Figure 4. Time-height cross-section of system-averaged quantities within a $3^{\circ} \times 3^{\circ}$ box from 00:00 UTC 30 August to 18:00 UTC 6 September. Relativity humidity is shown in (a), relative vorticity $(\zeta)$ with relative humidity contours of $40,50,60$, and $70 \%$ is shown in (b), and mass flux with relative humidity contours of 40, 50, 60, and $70 \%$ is shown in (c). A dry layer near and above $600 \mathrm{hPa}$ appears on 2 September and persists through 6 September. There are corresponding decreases in relative vorticity and mass flux at these times.

provide a first look into the existing flow topology around exGaston's pouch. Figure 5 shows the horizontal flow fields and calculated dividing streamlines at 18:00 UTC 2 September from ECMWF analysis data at 500, 700, 850, and $925 \mathrm{hPa}$ levels when dry air was greatly impacting the pouch.

At 700, 850, and $925 \mathrm{hPa}$, a hyperbolic point lies east of the circulation center. However, to the west of the circulation center, the pouch is open to the environment, thereby providing a pathway for air parcels to enter the pouch (i.e., an "open pouch"). At $500 \mathrm{hPa}$ the hyperbolic point is northwest of the circulation center, and the pouch is open to the east. This interpretation is consistent with the study of Rutherford and Montgomery (2012, hereafter referred to as RM12), who performed a more comprehensive study of the flow topology of ex-Gaston. In particular, RM12 analyzed Lagrangian coherent structures derived from particle trajectories, and found that lateral, dry air intrusion occurred from 1 to 5 September (RM12 Fig. 6 therein). These current findings at 700, 850, and $925 \mathrm{hPa}$ are consistent with the detailed Lagrangian analysis of ex-Gaston by RM12.

We can further study the structure of the pouch by looking at the time-dependent nature of the flow by calculating hyperbolic trajectories (Samelson and Wiggins, 2006). Hyperbolic trajectories are trajectories of the time-independent flow field that share the same linear stability properties as hyperbolic fixed points in time-independent flow. These hyperbolic trajectories have stable and unstable manifolds associated with them, and these manifolds control particle transport in timedependent flow (Ide et al., 2002).

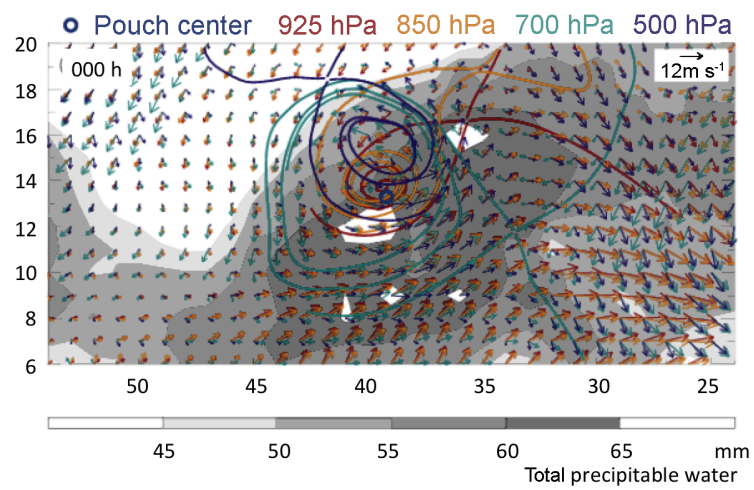

Figure 5. Dividing streamlines at 18:00 UTC 2 September 2010 are shown for $500,700,850$, and $925 \mathrm{hPa}$, and are overlaid on comoving wind vectors at each level and total precipitable water.

Figure 6 shows a time sequence of stable and unstable manifolds at $500 \mathrm{hPa}$ (left column) and $700 \mathrm{hPa}$ (right column) from 00:00 UTC 1 September to 00:00 UTC 3 September. Stable manifolds are indicated with red lines, and unstable manifolds are indicated with blue and cyan lines. For reference, a $3^{\circ}$ radius circle around ex-Gaston's diagnosed pouch center is indicated by the green circle. Throughout this $48 \mathrm{~h}$ period, the stable manifold (red line) and an unstable manifold (blue line) intersect east of ex-Gaston's pouch on the 500 and $700 \mathrm{hPa}$ pressure surfaces. The intersection of these manifolds marks the location of a hyperbolic trajectory, and the persistence of these manifolds is indicative of the pouch having a barrier to intrusions from the northeast, east and southeast. In this case the manifolds comprise only part of a cat's eye. At $700 \mathrm{hPa}$, the stable (red line) manifold also intersects the unstable (cyan line) manifold south of exGaston's pouch. We do not observe this second intersection on the $500 \mathrm{hPa}$ pressure level. At both the 500 and $700 \mathrm{hPa}$ levels, there are no intersecting manifolds west of the pouch. No intersection implies no additional hyperbolic trajectory, and leaves no way for boundaries to be topologically connected into a separatrix. ${ }^{3}$ While there is a difference between the dividing streamline and Lagrangian manifold analyses at $500 \mathrm{hPa}$, the two methods are consistent at the other pressure levels presented. The Lagrangian manifold method is more complete and accurate by incorporating the time-dependent nature of the analyzed flow.

To identify the source region for the dry air that entered exGaston's pouch at 700, 600, 500, and $400 \mathrm{hPa}$ on 18:00 UTC 2 September, backward trajectories were computed for particles seeded within a $3^{\circ}$ radius of the pouch center. Trajectories were computed as in RM12 using a fourth-order Runge-Kutta method with a $15 \mathrm{~min}$ intermediate time step and bi-cubic interpolation in both time and space on constant pressure surfaces. At $400 \mathrm{hPa}$, particles that are within a $3^{\circ}$

\footnotetext{
${ }^{3}$ A separatrix is a flow partitioning boundary formed by connected segments of manifolds or material curves.
} 

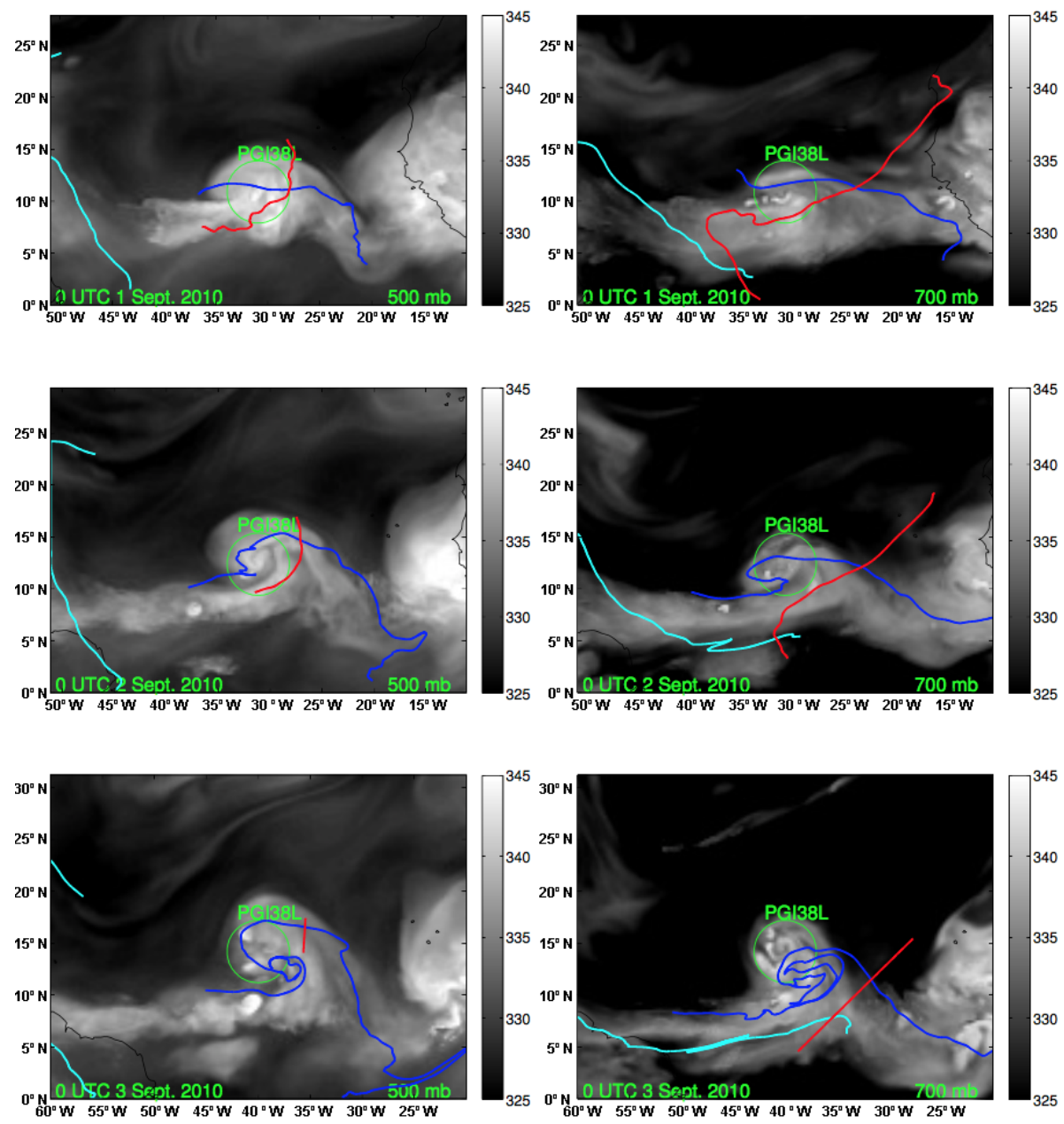

Figure 6. Lagrangian manifolds are overlaid on $\theta_{\mathrm{e}}$ fields at $500 \mathrm{hPa}$ (left column) and $700 \mathrm{hPa}$ (right column) from 1 to 3 September. Stable manifolds are red, and unstable manifolds are blue and cyan. The manifolds indicate that the pouch had a hyperbolic point to the east, but was open to environmental air to the west.

radius of the pouch at 18:00 UTC 2 September originated to the north of the pouch (Fig. 7). At the $500 \mathrm{hPa}$ level, particles that are within a $3^{\circ}$ radius of the pouch at 18:00 UTC 2 September originated primarily northeast and southwest of the pouch (Fig. 8).

For the trajectories identified in the foregoing figures, it is of interest to document the evolution of pseudo-equivalent potential temperature, $\theta_{\mathrm{e}}$. For a moist air parcel, $\theta_{\mathrm{e}}$ is approximately materially conserved in the absence of mixing processes. On a given pressure surface, $\theta_{\mathrm{e}}$ is a function of moisture and temperature and because of its tracer-like property and weak temperature gradient in the tropics, increases or decreases in $\theta_{\mathrm{e}}$ along a constant pressure trajectory reflect primarily changes in moisture. For all calculations presented here, we use the $\theta_{\mathrm{e}}$ definition as given by Bolton (1980) (his Eq. 43).

Figure 9 summarizes the evolution of $\theta_{\mathrm{e}}$ for the trajectories identified previously in Fig. 8 on the $500 \mathrm{hPa}$ level. A colored point in the figure represents a snapshot of the particular particle's $\theta_{\mathrm{e}}$ and radial distance from the center of Gaston's pouch. The colors range from brown to blue, with brown denoting the earliest time of 00:00 UTC 31 August and blue denoting the latest time of 18:00 UTC 2 September. The quasi-regular pattern of blue dots between 0 and $3^{\circ}$ radius is a manifestation of the initial seeding method for the backward trajectory calculation.

Figure 9 shows that particles seeded within the nominal pouch radius of $3^{\circ}$ originate from two distinct source regions (brown points) outside of the pouch. The two source regions are indicated by the red dots in Fig. 8; one source region is located in an arch-like filament northeast of the pouch in a dry (low $\theta_{\mathrm{e}} \sim 328 \mathrm{~K}$ ) environment; the other is located west and southwest of the pouch in a relatively moist environment $\left(\theta_{\mathrm{e}} \sim 339 \mathrm{~K}\right)$. As these particles enter ex-Gaston's pouch from 00:00 UTC 31 August to 18:00 UTC 2 September, the moist particles remain relatively moist, and the dry particles remain relatively dry. The black slanted line in Fig. 9 approximately differentiates these moist and dry trajectory 


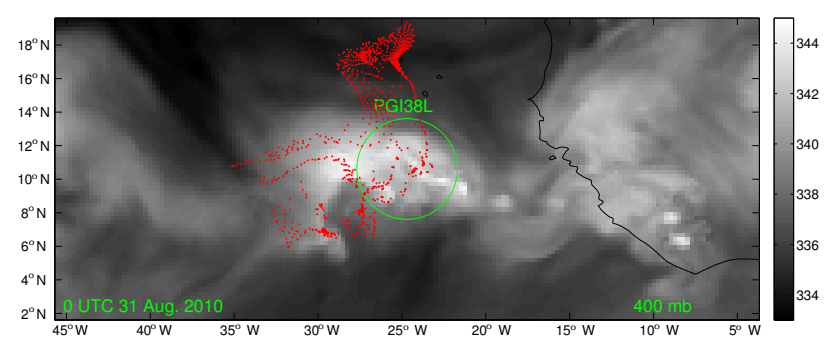

Figure 7. Particle trajectory locations at $400 \mathrm{hPa}$ 18:00 UTC $31 \mathrm{Au}-$ gust are overlaid on $\theta_{\mathrm{e}}(\mathrm{K})$ valid at 00:00 UTC 31 August 2010. These trajectories are all within a radius of $3^{\circ}$ of the pouch center (green circle) by 18:00 UTC 2 September.

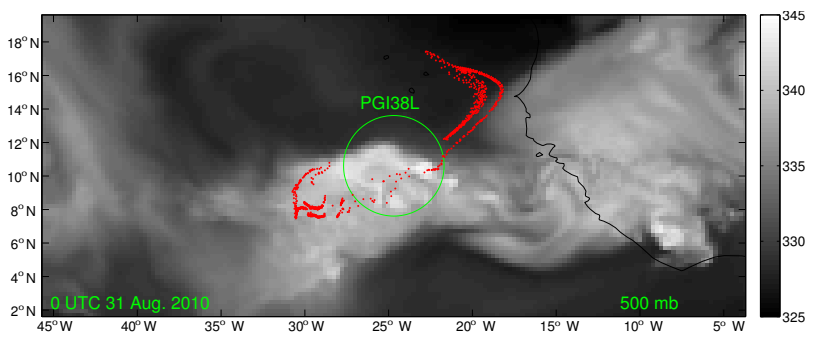

Figure 8. Particle trajectory locations at $500 \mathrm{hPa}$ 18:00 UTC $31 \mathrm{Au}-$ gust are overlaid on $\theta_{\mathrm{e}}(\mathrm{K})$ 00:00 UTC 31 August 2010. These trajectories are all within a radius of $3^{\circ}$ of the pouch center (green circle) by 18:00 UTC 2 September.

paths, and its shallow slope indicates that dry air was not significantly moistened before entering the pouch. A similar analysis was performed for the $400 \mathrm{hPa}$ level (not shown), and showed similar trends as the $500 \mathrm{hPa}$ level. These results demonstrate that dry air was entering Gaston's pouch during this $66 \mathrm{~h}$ period from 00:00 UTC 31 August to 18:00 UTC 2 September.

\section{Implications of dry air and a degraded pouch}

The findings from the previous section showing dry air entering ex-Gaston's pouch motivate an important question in its non-redevelopment: what was the role of dry air in the non-development? To understand the role of the dry air entering the pouch it is useful to review previous studies of the role of dry air on convection. A new hypothesis on the role of dry air in tropical cyclogenesis was inspired in part by the work of Smith and Montgomery (2012). The authors studied the convective environments of the tropical disturbances during the PREDICT experiment. They found that a prominent difference between developing and non-developing disturbances was the difference in $\theta_{\mathrm{e}}$ between the surface and $3 \mathrm{~km}$. Smith and Montgomery (2012) hypothesized that entrained, dry air weakens the convective updraughts and thereby weakens the vortex-tube stretching of ambient and local cyclonic vorticity. Weakening of the convective updraughts implies

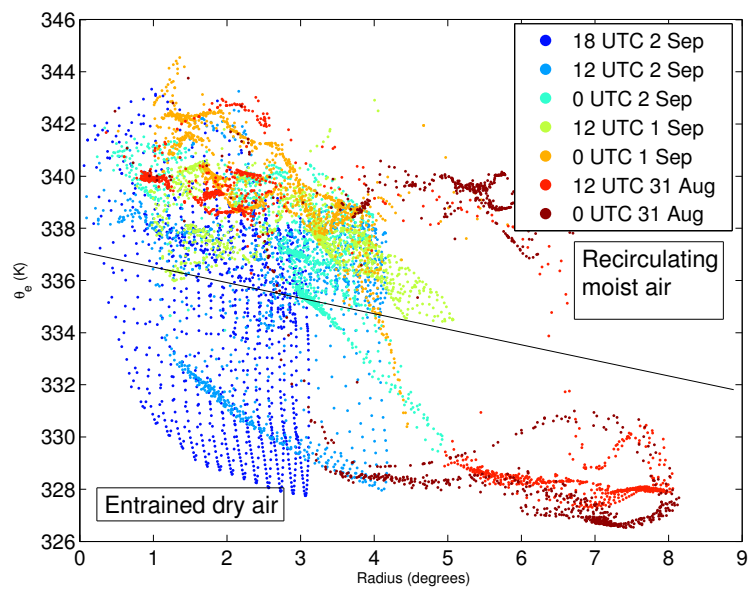

Figure 9. $\theta_{\mathrm{e}}$ and radial distance from the center of Gaston's pouch at the $500 \mathrm{hPa}$ level. The colors range from brown to blue, with brown denoting the earliest time of 00:00 UTC 31 August and blue denoting the latest time of 18:00 UTC 2 September.

a frustrated vorticity amplification process. The hypothesis of Smith and Montgomery (2012) stands in contrast to the traditional notion that dry air increases the strength of convective downdraughts and increases the low-level divergence that accompanies these downdraughts.

In another study of convective environments, James and Markowski (2010) investigated the role of dry air aloft on deep convection. In their numerical study, they found that in the low CAPE environments $\left(1500 \mathrm{~J} \mathrm{~kg}^{-1}\right)$ with a dry air layer of $\mathrm{RH}=70 \%$ near $700 \mathrm{hPa}$, the updraught mass flux was reduced throughout the depth of the troposphere, and the downdraught mass flux was either unchanged or reduced.

In their numerical study of rotating convection during tropical cyclogenesis, Kilroy and Smith (2012) (hereafter referred to as KS12) investigated the role of the dry air. KS12 created an idealized sounding based on the ex-Gaston environment, and proceeded to modify the idealized sounding by injecting dry air into the mid-levels. They found through a series of experiments (summarized in their Table 2), that dry air aloft reduced the convective updraught strength and the vertical extent of the convective updraught.

KS12 also used a "moist" and a "dry" profile from the PREDICT Experiment. The moist profile was from 18:20 UTC 5 September, and had a total precipitable water (TPW) of $65.2 \mathrm{~kg} \mathrm{~m}^{-2}$. The dry profile was from 14:48 UTC 5 September, and TPW $=43.5 \mathrm{~kg} \mathrm{~m}^{-2}$. In the moist environment, KS12 found maximum convective updraught and downdraught velocities of 34 and $10.9 \mathrm{~m} \mathrm{~s}^{-1}$, respectively, and vertical extent above $10 \mathrm{~km}$. However, in the dry environment the maximum updraught velocity and downdraught velocities were 11.4 and $6.3 \mathrm{~m} \mathrm{~s}^{-1}$, respectively, and the vertical extent was only $\sim 7 \mathrm{~km}$ (see KS12 Fig. 7). Dry air reduced both the updraught strength and the maximum vertical extent, while the downdraught velocities were only moder- 
Table 1. Summary of dropsondes from PREDICT Research Flight 9 (RF09) on 2 September.

\begin{tabular}{lrrrr}
\hline Drop num. & $\begin{array}{r}\text { Time } \\
(\mathrm{UTC})\end{array}$ & $\begin{array}{r}\text { TPW } \\
\left(\mathrm{kg} \mathrm{m}^{-2}\right)\end{array}$ & $\begin{array}{r}\text { CAPE } \\
\left(\mathrm{J} \mathrm{kg}^{-1}\right)\end{array}$ & $\begin{array}{r}\mathrm{CIN} \\
\left(\mathrm{J} \mathrm{kg}^{-1}\right)\end{array}$ \\
\hline 1 & $15: 32$ & 33.0 & 478 & 149 \\
2 & $15: 44$ & 48.2 & 196 & 95 \\
3 & $15: 55$ & 53.6 & 24 & 142 \\
4 & $16: 05$ & 61.1 & 688 & 6 \\
5 & $16: 14$ & 62.8 & 706 & 0 \\
$6^{*}$ & $16: 24$ & 57.5 & 1047 & 9 \\
$7^{*}$ & $16: 37$ & 58.4 & 612 & 29 \\
$8^{*}$ & $16: 47$ & 63.0 & 1707 & 0 \\
$9^{*}$ & $16: 54$ & 65.9 & 654 & 11 \\
$10^{*}$ & $17: 03$ & 67.1 & 1649 & 0 \\
$11^{*}$ & $17: 13$ & 59.9 & 1566 & 0 \\
$12^{*}$ & $17: 23$ & 57.5 & 605 & 5 \\
13 & $17: 33$ & 56.7 & 2 & 158 \\
14 & $17: 45$ & 55.3 & 0 & 328 \\
15 & $17: 55$ & 53.8 & 114 & 110 \\
16 & $18: 08$ & 51.1 & 1155 & 14 \\
17 & $18: 18$ & 35.6 & 525 & 75 \\
18 & $18: 30$ & 36.1 & 285 & 143 \\
19 & $18: 43$ & 38.2 & 101 & 155 \\
\hline
\end{tabular}

* denotes dropsonde is in the pouch-scale analysis here. Adapted from Smith and Montgomery (2012).

ately reduced, consistent with findings from the experiments with the idealized soundings. These results showed that dry air reduces cloud buoyancy, thus making mass flux profiles weaker and shallower than in a moist environment, as well as making the convective updraught less effective in amplifying vertical vorticity (Smith and Montgomery, 2012).

We examined the dropsonde data from 2 September, and compared the profiles to the data used by KS12. We found profiles from within the pouch on 2 September with similar characteristics as the profiles used by KS12. This detailed comparison is not shown. However Table 1 shows TPW, CAPE, and CIN from dropsondes on 2 September (see Smith and Montgomery, 2012 for a thorough analysis of the PREDICT thermodynamic data). The 2 September data compare well with the profiles used by KS12.

In total, the foregoing results suggest that the pouch was vulnerable to the environment with dry air penetrating the pouch and disrupting the amplification of vorticity. This analysis of the kinematic, dynamic, and thermodynamic structure of ex-Gaston in the ECMWF analysis, as well as the work of RM12 and Davis and Ahijevych (2012, Fig. 9 therein), show that ex-Gaston's pouch was misaligned, and vulnerable to environmental air as early as 2 September (Fig. 4), the day of the first PREDICT research flight into this remnant low. Based on the findings of KS12, convective updraughts that form in this region containing dry air aloft would be expected to result in divergence near the $600 \mathrm{hPa}$ level, thus causing an expanding material loop at these levels. From Kelvin's cir- culation theorem, as the material loop expands the absolute vertical vorticity must decrease in order to conserve absolute circulation. A reduction in vorticity will create a compromised pouch, which will allow further intrusion of dry air and inhibit vorticity amplification.

Gjorgjievska and Raymond (2014, hereafter referred to as GR14) propose a different process that leads to the failed redevelopment of ex-Gaston. GR14 (p. 3076) hypothesize that the "severe decrease of the mid-level vortex observed between the period of Gaston 1 (2 September) and Gaston 2 (3 September) was a deciding factor for Gaston's failure to re-intensify". It is important to note that GR14, RM12, and Davis and Ahijevych (2012) agree on the decay of the midlevel vortex. GR14 hypothesize that convection was suppressed by a strong trade wind inversion, and attribute the decrease in the mid-level vorticity to this strong trade inversion and corresponding structure of the vertical mass flux profile. GR14 argue that the trade wind inversion air causes the decrease in magnitude with height of the mass flux profile. However, we contend that the intrusion of dry air at and above the $600 \mathrm{hPa}$ level is responsible for the decrease in the mass flux profile.

To address the hypothesized influence of the strong trade wind inversion, it proves useful to review the thermodynamic structure of the dropsonde data collected on 2 September. The PREDICT experiment released 19 dropsondes in exGaston on this day. GR14 included drop numbers 2 through 14 (see Table 1) in their 3DVAR analysis and area-averaging schemes. Within their $4^{\circ} \times 4^{\circ}$ analysis box (their Fig. 8), only one drop (drop number 2, located northwest of exGaston's pouch) shows clear evidence of a temperature inversion (Fig. 10), while 11 of the profiles show evidence of a dry layer above $600 \mathrm{hPa}$, not shown. It is unclear how one particular sounding could have such a hypothesized impact on the system-scale vorticity dynamics.

Our study of GR14 suggests that these authors appeared to overlook the implications of Davis and Ahijevych (2012) findings of a vertically sheared pouch and RM12's findings of dry air mixing into ex-Gaston's pouch between 1 and 3 September (RM12's Fig. 6), a time period spanning the first day of PREDICT observations (2 September). While GR14 acknowledge the role of a time-dependent or transient flow component in causing a reversal in the sign of the low-level vorticity tendency, they appear to not recognize that this same time-dependence can cause an intrusion of dry air to enter a pouch that is apparently closed in an instantaneous snapshot. Thus, GR14 appear also to misinterpret the results of Smith and Montgomery (2012) and RM12: GR14 (p. 3076-3077) imply that ex-Gaston's pouch was "robust" (i.e., closed) on 2 September, and therefore unlikely that "dry air might have been drawn into the core of Gaston".

Our offered hypothesis of Gaston's non-redevelopment described above stands somewhat in contrast to the alternative hypothesis by Gjorgjievska and Raymond (2014). The data shown herein support the view that dry air penetrated the 

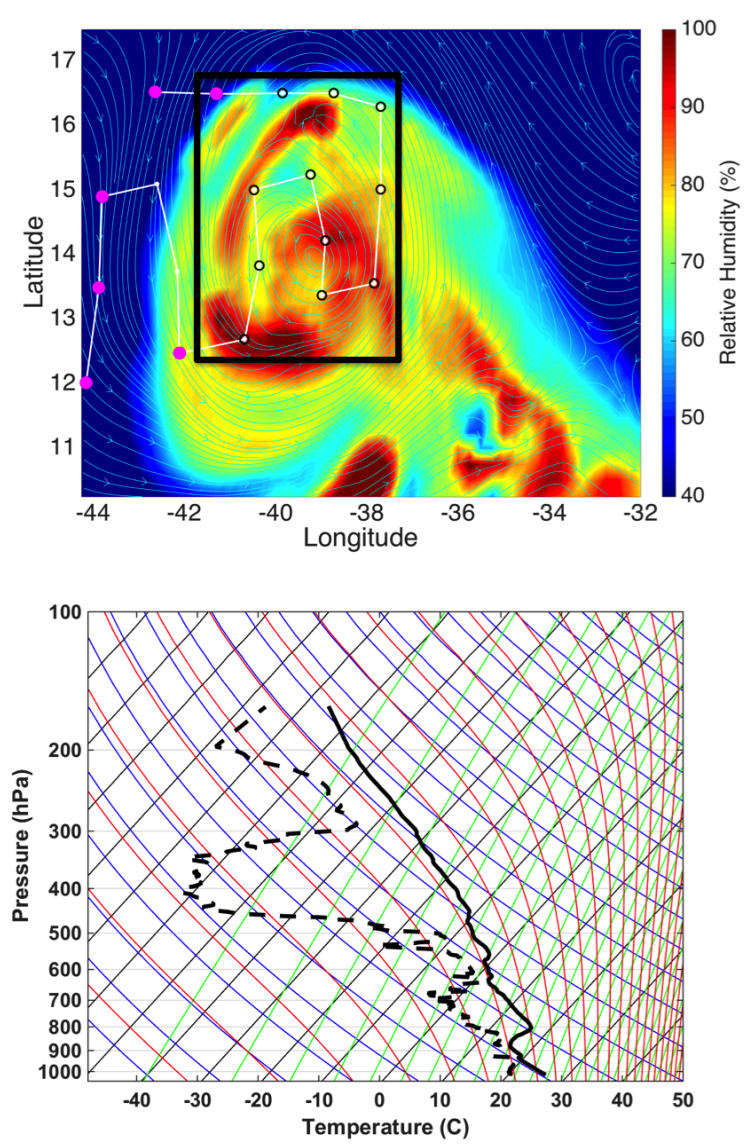

Figure 10. PREDICT flight path (white line) and dropsonde locations (dots) overlaid on $700 \mathrm{hPa}$ relative humidity (shading) and co-moving streamlines (cyan lines) from ECMWF analysis data at 18:00 UTC 2 September (top panel). The magenta dots indicate locations where dropsonde data show evidence of an inversion. The black box corresponds to GR14 Fig. 8. Only one of the 12 soundings in the GR14 area of interest shows evidence of an inversion (bottom panel).

pouch before the first flight into ex-Gaston and disrupted the amplification of vorticity at those levels where dry air intruded. GR14 agree with this hypothesis of a compromised pouch and dry air intrusion, but only after 4 September.

The data from PREDICT research flight 9 on 2 September do show evidence for an inversion outside the GR14 analysis box for ex-Gaston's pouch (Fig. 10). To investigate the influence of the dry, trade inversion air west of ex-Gaston's pouch on 2 September, we performed a forward trajectory analysis (Fig. 11). Particles were seeded on the $850 \mathrm{hPa}$ pressure level west of the sweet spot location (where the PREDICT data show a temperature inversion) at 12:00 UTC 2 September and integrated forward to 00:00 UTC 4 September. Nearly all of the particles are located outside of a $3^{\circ}$ radius of the pouch center by 00:00 UTC 4 September. This analysis shows that the observed dry, trade inversion air on 2 September does

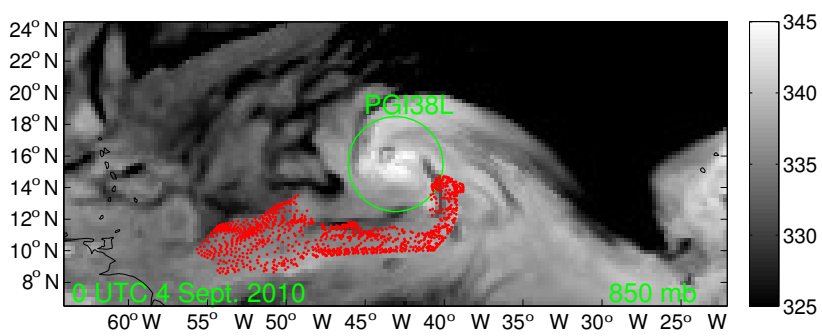

Figure 11. Particle trajectory locations at $850 \mathrm{hPa}$ at 00:00 UTC 4 September overlaid on $\theta_{\mathrm{e}}(\mathrm{K})$. Particles were seeded west of exGaston's pouch at 12:00 UTC 2 September, within a suspected trade wind inversion layer. These trajectories are all outside of a $3^{\circ}$ radius of the pouch center (green circle) on 4 September.

not enter the pouch, and has little influence on the nondevelopment of ex-Gaston.

\section{Conclusions}

Our study of the ECMWF analysis data demonstrates that ex-Gaston did not have a robust pouch and was open to the intrusion of environmental air at the mid- to upper levels on all days of the aircraft observations. Ex-Gaston's pouch was closed to dry air intrusion at low levels. Lagrangian trajectory and manifold calculations using ECMWF analyses show that dry air did indeed penetrate the pouch. These findings support the hypothesis that entrained, dry air near $600 \mathrm{hPa}$ inhibited convective updraughts and vertical mass flux, which in turn leads to a reduction in vorticity and a compromised pouch at these middle levels. A compromised pouch allows further intrusion of dry air and inhibits subsequent vorticity amplification, as described in the work of Smith and Montgomery (2012). The findings presented herein support our hypothesis that ex-Gaston's degraded pouch further led to the non-redevelopment of the system by limiting the amplification of vorticity and not providing a protected environment for sufficient vorticity aggregation, consistent with the marsupial paradigm of tropical cyclogenesis described by DMW09.

\section{Data availability}

All analysis data used for this paper are available by contacting the corresponding author of this manuscript via email.

Acknowledgements. T. M. Freismuth acknowledges OPNAV N2/N6, CNMOC, and valuable discussions with Gerard Kilroy and Tim Dunkerton. B. Rutherford acknowledges the support of NSF AGS-1432983. M. T. Montgomery acknowledges the support of NSF AGS-1313948, NOAA HFIP grant N0017315WR00048, NASA grant NNG11PK021 and the US Naval Postgraduate School. ECMWF data provided by Peter Bauer at ECMWF, 
Gerald Thomsen, and Gerard Kilroy and Roger Smith from the Ludwig Maximilian University of Munich and the Deutscher Wetterdienst. The views expressed herein are those of the authors and do not represent sponsoring agencies or institutions.

Edited by: T. J. Dunkerton

\section{References}

Bolton, D.: The computation of equivalent potential temperature, Mon. Weather Rev., 108, 1046-1053, doi:10.1175/15200493(1980)108<1046:TCOEPT>2.0.CO;2, 1980.

Davis, C. A. and Ahijevych, D. A.: Mesoscale structural evolution of three tropical weather systems observed during PREDICT, J. Atmos. Sci., 69, 1284-1305, doi:10.1175/JAS-D-110225.1, 2012.

Davis, C. A. and Ahijevych, D. A.: Thermodynamic environments of deep convection in atlantic tropical disturbances, J. Atmos. Sci., 70, 1912-1928, doi:10.1175/JAS-D-12-0278.1, 2013.

Dunkerton, T. J., Montgomery, M. T., and Wang, Z.: Tropical cyclogenesis in a tropical wave critical layer: easterly waves, Atmos. Chem. Phys., 9, 5587-5646, doi:10.5194/acp-9-5587-2009, 2009.

Gjorgjievska, S. and Raymond, D. J.: Interaction between dynamics and thermodynamics during tropical cyclogenesis, Atmos. Chem. Phys., 14, 3065-3082, doi:10.5194/acp-14-3065-2014, 2014.

Haynes, P. H. and McIntyre, M. E.: On the evolution of vorticity and potential vorticity in the presence of diabatic heating and frictional or other forces, J. Atmos. Sci., 44, 828-841, doi:10.1175/1520-0469(1987)044<0828:OTEOVA>2.0.CO;2, 1987.

Ide, K., Small, D., and Wiggins, S.: Distinguished hyperbolic trajectories in time-dependent fluid flows: analytical and computational approach for velocity fields defined as data sets, Nonlin. Processes Geophys., 9, 237-263, doi:10.5194/npg-9-237-2002, 2002.

James, R. P. and Markowski, P. M.: A numerical investigation of the effects of dry air aloft on deep convection, Mon. Weather Rev., 138, 140-161, doi:10.1175/2009MWR3018.1, 2010.

Kilroy, G. and Smith, R. K.: A numerical study of rotating convection during tropical cyclogenesis, Q. J. Roy. Meteor. Soc., 139, 1255-1269, doi:10.1002/qj.2022, 2012.

Lussier III, L.: A multi-scale analysis of the tropical cyclogenesis within the critical layer of tropical easterly waves in the Atlantic and Western North Pacific sectors, PhD thesis, Naval Postgraduate School, Monterey, CA, USA, 2010.

Lussier III, L. L., Montgomery, M. T., and Bell, M. M.: The genesis of Typhoon Nuri as observed during the Tropical Cyclone Structure 2008 (TCS-08) field experiment - Part 3: Dynamics of low-level spin-up during the genesis, Atmos. Chem. Phys., 14, 8795-8812, doi:10.5194/acp-14-8795-2014, 2014.

Lussier III, L. L., Rutherford, B., Montgomery, M., and Boothe, M.: Examining the roles of the easterly wave critical layer and vorticity accretion during the tropical cyclogenesis of Hurricane Sandy, Mon. Weather Rev., 143, 1703-1722, doi:10.1175/MWR-D-1400001.1, 2015.
Montgomery, M. T., Wang, Z., and Dunkerton, T. J.: Coarse, intermediate and high resolution numerical simulations of the transition of a tropical wave critical layer to a tropical storm, Atmos. Chem. Phys., 10, 10803-10827, doi:10.5194/acp-1010803-2010, 2010a.

Montgomery, M. T., Lussier III, L. L., Moore, R. W., and Wang, Z.: The genesis of Typhoon Nuri as observed during the Tropical Cyclone Structure 2008 (TCS-08) field experiment - Part 1: The role of the easterly wave critical layer, Atmos. Chem. Phys., 10, 9879-9900, doi:10.5194/acp-10-9879-2010, 2010 b.

Montgomery, M. T., Davis, C., Dunkerton, T., Wang, Z., Velden, C., Torn, R., Majumdar, S. J., Zhang, F., Smith, R. K., Bosart, L., Bell, M. M., Haase, J. S., Heymsfield, A., Jensen, J., Campos, T., and Boothe, M. A.: The pre-depression investigation of cloudsystems in the tropics (PREDICT) experiment: scientific basis, new analysis tools, and some first results, B. Am. Meteorol. Soc., 93, 153-172, doi:10.1175/BAMS-D-11-00046.1, 2012.

Nicholls, M. E. and Montgomery, M. T.: An examination of two pathways to tropical cyclogenesis occurring in idealized simulations with a cloud-resolving numerical model, Atmos. Chem. Phys., 13, 5999-6022, doi:10.5194/acp-13-5999-2013, 2013.

Raymond, D. J. and López Carrillo, C.: The vorticity budget of developing typhoon Nuri (2008), Atmos. Chem. Phys., 11, 147163, doi:10.5194/acp-11-147-2011, 2011.

Raymond, D. J., Gjorgjievska, S., Sessions, S., and Fuchs, Ž.: Tropical cyclogenesis and mid-level vorticity, Aust. Met. Ocean. Soc. J., 64, 11-25, 2014.

Reasor, P. D., Montgomery, M. T., and Grasso, L. D.: A new look at the problem of tropical cyclones in vertical shear flow: Vortex resiliency, J. Atmos. Sci., 61, 3-22, doi:10.1175/15200469(2004)061<0003:ANLATP>2.0.CO;2, 2004.

Riemer, M. and Montgomery, M. T.: Simple kinematic models for the environmental interaction of tropical cyclones in vertical wind shear, Atmos. Chem. Phys., 11, 9395-9414, doi:10.5194/acp-11-9395-2011, 2011.

Rutherford, B. and Montgomery, M. T.: A Lagrangian analysis of a developing and non-developing disturbance observed during the PREDICT experiment, Atmos. Chem. Phys., 12, 11355-11381, doi:10.5194/acp-12-11355-2012, 2012.

Samelson, R. M. and Wiggins, S.: Lagrangian Transport in Geophysical Jets and Waves: The Dynamical Systems Approach, Vol. 31, Springer Science \& Business Media, Berlin, Germany, 2006.

Smith, R. K. and Montgomery, M. T.: Observations of the convective environment in developing and non-developing tropical disturbances, Q. J. Roy. Meteor. Soc., 138, 1721-1739, doi:10.1002/qj.1910, 2012.

Wang, Z., Montgomery, M. T., and Dunkerton, T. J.: Genesis of PreHurricane Felix (2007). Part II: Warm core formation, precipitation evolution, and predictability, J. Atmos. Sci., 67, 1730-1744, doi:10.1175/2010JAS3435.1, 2010a.

Wang, Z., Montgomery, M. T., and Dunkerton, T. J.: Genesis of Pre-Hurricane Felix (2007). Part I: The role of the easterly wave critical layer, J. Atmos. Sci., 67, 1711-1729, doi:10.1175/2009JAS3420.1, 2010b. 